\title{
New 2D Lanthanide MOFs Constructed from Bis(imide) Pyromellitic Alanine Ligands with Enhanced Fluorescence toward Activation and Modulation of Microstructure
}

Fernando González Chávez, ${ }^{[1]}$ Hugo Nájera, ${ }^{[2]}$ Marco Antonio Leyva, ${ }^{[3]}$ Omar Solorza-Feria, ${ }^{[3]}$ Federico González, ${ }^{[4]}$ and Hiram Isaac Beltrán. ${ }^{*[5]}$

\section{Supporting Information}

\section{Table of contents}

Spectroscopic and physicochemical characterization ...................................................... 2

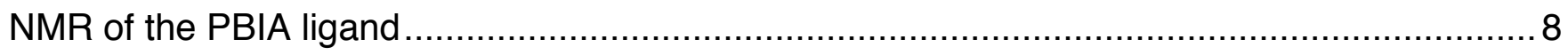

FTIR

Comparison between inactivated and activated materials. ............................................. 9

Single crystal X-ray selected geometrical parameters...................................................... 10

Ortep plots of PBIA-Ln single crystal structures (asymmetric unit) ................................... 12

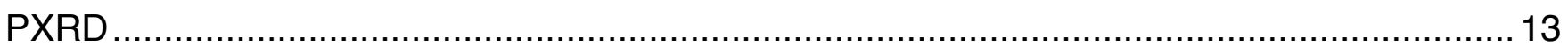

Comparison between simulated (by single crystal) and experimental X-ray powder patterns. 13

Comparison between X-ray diffractograms of inactivated and activated materials............... 14

TGA

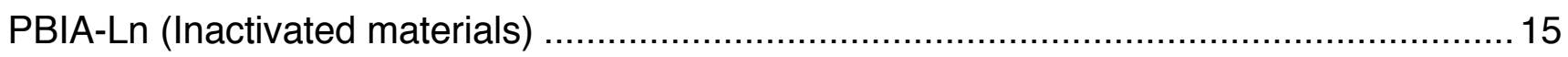

Comparison between activated and inactivated materials .............................................. 18 


\section{Spectroscopic and physicochemical characterization}

\section{2,2'-(1,3,5,7-tetraoxo-5,7-dihydropyrrolo[3,4-f]isoindole-2,6(1H,3H)-diyl)dipropionic}

(PBIA). White solid, 42\% yield. ${ }^{1} \mathrm{H}$ NMR $\left(60 \mathrm{MHz}, \delta=\mathrm{ppm}, \mathrm{DMSO}-d_{6}\right): 8.26$ (s, H-6, 2H), 4.90 (c, H-2, $\mathrm{J} O=7.2 \mathrm{~Hz}, 2 \mathrm{H}), 3.7\left(\mathrm{H}_{2} \mathrm{O}\right), 1.57(\mathrm{~d}, \mathrm{H}-3, \mathrm{~J} O=7.2 \mathrm{~Hz}, 6 \mathrm{H}) . \mathrm{D}_{2} \mathrm{O}$ drops were added to remove a broad signal from the labile proton of the carboxylic acid that prevented an adequate integration of the signal in 4.90 ppm. RMN ${ }^{13} \mathrm{C}(15 \mathrm{MHz}, \delta=$ ppm, DMSO-d $)$ : 170.7 (C-1, 2C), 165.5 (C-4, 4C), $136.9(\mathrm{C}-6,2 \mathrm{C}), 118.0$ (C-5, 4C), 43.8 (C-2, 2C), 14.3 (C3, 2C). FTIR (v, $\mathrm{cm}^{-1}$, KBr pellet): 3600-2700 (O-H), 3098, 3066, 3036, $3000\left(\mathrm{C}_{\mathrm{sp} 2}-\mathrm{H}\right), 2953,2920\left(\mathrm{C}_{\mathrm{sp} 3}-\mathrm{H}\right), 1780,1717\left(-\mathrm{C}=\mathrm{O}-\mathrm{N}-\mathrm{C}=\mathrm{O}-, \mathrm{C}=\mathrm{O}_{\mathrm{COOH}}\right), 1468,1455 \delta_{\text {asym }}\left(\mathrm{C}_{\mathrm{sp} 3}-\mathrm{H}\right)$, $1384\left(\mathrm{C}-\mathrm{N}_{\text {imide }}\right), 1365 \delta_{\mathrm{sym}}\left(\mathrm{C}_{\mathrm{sp} 3}-\mathrm{H}\right), 1296,1252,1168,1155,1087,1078,1023$ (C-O), 946, 923, 852, 817, 768, 734 (C-N-C out of plane bending), 670, 617, 566, 460, 427, 406. PXRD ( ${ }^{\circ}, 2 \theta$ (count number)): 14.3 (70.9), 15.3 (95.4), 15.5 (80.3), 16.8 (100.1), 16.9 (101.3), 18.18 (164.0), 19.5 (204.2), 21.0 (133.6), 23.0 (79.2), 26.6 (95.1), 26.8 (80.9), 28.1 (37.6), 29.3 (74.4), 31.2 (68.8). TGA (\% wt. loss, $\left.\mathrm{T}_{\text {interval }}\left[{ }^{\circ} \mathrm{C}\right]\right): 50$, 20-416; 46, 416-982.

PBIA-Pr. Light green solid, 32.8\% yield. FTIR (v, $\mathrm{cm}^{-1}$, KBr pellet): 3600-2700 (O-H, $\left.\mathrm{H}_{2} \mathrm{O}_{\text {atm }}\right), 3100$, 3074, $3041\left(\mathrm{C}_{\mathrm{sp} 2}-\mathrm{H}\right), 2997,2944\left(\mathrm{C}_{\mathrm{sp} 3}-\mathrm{H}\right), 1773,1720(-\mathrm{C}=\mathrm{O}-\mathrm{N}-\mathrm{C}=\mathrm{O}-), 1656 v_{\mathrm{asym}}\left(\mathrm{COO}_{\text {mono }}\right), 1631$ $\left(\mathrm{C}=\mathrm{O}_{\mathrm{DMF}} \rightarrow \mathrm{Pr}\right), 1604 v_{\text {asym }}\left(\mathrm{COO}_{\text {aniso }}\right), 1582 v_{\text {asym }}\left(\mathrm{COO}_{\text {iso }}\right), 1499,1461 \delta_{\text {asym }}\left(\mathrm{C}_{\text {sp } 3}-\mathrm{H}\right), 1418 v_{\text {sym }}(\mathrm{COO})$, $1386\left(\mathrm{C}-\mathrm{N}_{\text {imide }}\right), 1362 \delta_{\text {sym }}\left(\mathrm{C}_{\mathrm{sp} 3}-\mathrm{H}\right), 1346 v_{\text {sym }}(\mathrm{COO}), 1285,1253,1174,1158,1134,1108,1082(\mathrm{C}-\mathrm{O})$, 1023, 931, 864, 838, 803, 784, 770, 733 (C-N-C out of plane bending) 677, 628, 565, 474, 414. PXRD ( ${ }^{\circ}$,

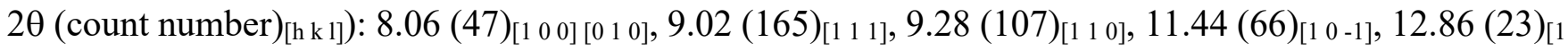

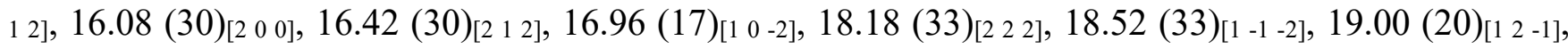

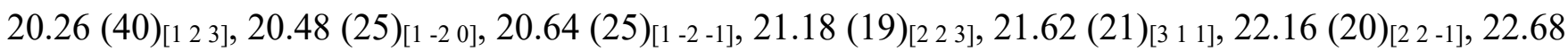

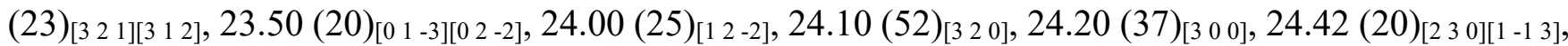

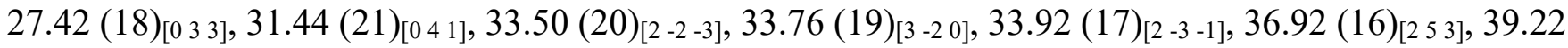


$\left.(17)_{[1} 36\right], 40.88(17)_{[1-3-5]}$. TGA (\% wt. loss, $\left.T_{\text {interval }}\left[{ }^{\circ} \mathrm{C}\right]\right): 2,20-104 ; 10,104-220 ; 8,220-375 ; 58,375-$ 995. Besides, light green single crystals suitable for X-ray diffraction analysis were obtained straight from the reaction vial and were diffracted as such.

PBIA-Pr ${ }_{\text {ACt. }}$ Light green solid. FTIR ( $v, \mathrm{~cm}^{-1}, \mathrm{KBr}$ pellet): 3600-2700 (O-H, $\left.\mathrm{H}_{2} \mathrm{O}_{\text {atm }}\right), 3100,\left(\mathrm{C}_{\mathrm{sp} 2}-\mathrm{H}\right)$, 2998, $2943\left(\mathrm{C}_{\mathrm{sp} 3}-\mathrm{H}\right), 1773,1716(-\mathrm{C}=\mathrm{O}-\mathrm{N}-\mathrm{C}=\mathrm{O}-), 1656 v_{\text {asym }}\left(\mathrm{COO}_{\text {mono }}\right) 1631\left(\mathrm{C}=\mathrm{O}_{\mathrm{DMF}} \rightarrow \operatorname{Pr}\right), 1582$ $v_{\text {asym }}\left(\mathrm{COO}_{\text {iso }}\right), 1499,1461 \delta_{\text {asym }}\left(\mathrm{C}_{\text {sp } 3-H}\right), 1419 v_{\text {sym }}(\mathrm{COO}), 1387\left(\mathrm{C}-\mathrm{N}_{\text {imide }}\right), 1366 \delta_{\text {sym }}\left(\mathrm{C}_{\text {sp } 3}-\mathrm{H}\right), 1343$ $v_{\text {sym }}(\mathrm{COO}), 1283,1253,1172,1157,1133,1108,1083$ (C-O), 1023, 933, 875, 839, 803, 771, 731 (C-NC out of plane bending) 676, 629, 565, 409. XRD ( ${ }^{\circ}, 2 \theta$ (count number)): 7.42 (124), 7.81 (489), 8.5 (449), 9.09 (80), 10.08 (71), 11.02 (128), 11.84 (103), 12.68 (126), 13.02 (153), 13.98 (167), 14.33 (115), 14.84 (144), 15.63 (176), 16.98 (121), 18.55 (96), 19.35 (154), 19.88 (209), 21.10 (230), 22.33 (351), 23.73 (172), 25.12 (192), 27.82 (99), 28.84 (66), 30.50 (101), 31.58 (110), 33.34 (101), 34.41 (102), $35.16(104)$, 36.95 (123). TGA (\% wt. loss, $\left.\mathrm{T}_{\text {interval }}\left[{ }^{\circ} \mathrm{C}\right]\right): 2,20-161 ; 9,161-355 ; 64,355-995$.

PBIA-Eu. Light green solid, 39\% yield. FTIR ( $v, \mathrm{~cm}^{-1}, \mathrm{KBr}$ pellet): 3600-2700 (O-H, $\left.\mathrm{H}_{2} \mathrm{O}_{\mathrm{atm}}\right), 3100$, 3074, $3042\left(\mathrm{C}_{\mathrm{sp} 2}-\mathrm{H}\right), 2998,2945\left(\mathrm{C}_{\mathrm{sp} 3}-\mathrm{H}\right)$, 1773, $1720(-\mathrm{C}=\mathrm{O}-\mathrm{N}-\mathrm{C}=\mathrm{O}-), 1657$ vasym $\left(\mathrm{COO}_{\text {mono }}\right), 1638$ $\left(\mathrm{C}=\mathrm{O}_{\mathrm{DMF}} \rightarrow \mathrm{Eu}\right), 1613 v_{\text {asym }}\left(\mathrm{COO}_{\text {aniso }}\right), 1588 v_{\text {asym }}\left(\mathrm{COO}_{\text {iso }}\right) \quad 1499,1462 \delta_{\text {asym }}\left(\mathrm{C}_{\mathrm{sp} 3}-\mathrm{H}\right), 1421 v_{\text {sym }}(\mathrm{COO})$, 1386 (C- Nimide), $1362 \delta_{\text {sym }}\left(\mathrm{C}_{\text {sp } 3}-\mathrm{H}\right), 1343 v_{\text {sym }}(\mathrm{COO}), 1285,1253,1175,1158,1134,1108,1082$ (C-O), $1024,932,864,838,803,784,770,733$ (C-N-C out of plane bending), 679, 628, 564, 477, 413. PXRD

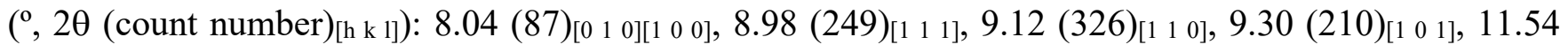

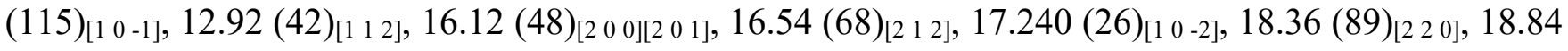
$\left.\left.(53)_{[12-1]}, 20.38(65)_{[1-2 ~ 0]}, 20.72(47)_{[1-2-1]}, 21.64(64)_{[3} 11\right], 22.76(56)_{[32 ~ 2]}, 23.48(37)_{[2} 1-2\right], 23.98(43)_{[3}$ $20], 24.28(53)_{[30} 00,25.78(31)_{[3} 3$ 2] $, 28.06(36)_{[32-1]}, 33.92(34)_{[2-3-1]}$. TGA (\% wt. loss, $\left.T_{\text {interval }}\left[{ }^{\circ} \mathrm{C}\right]\right): 3$, 
$20-115 ; 9,115-210 ; 9,210-360 ; 59,360-995$. Besides, light green single crystals suitable for X-ray diffraction analysis were obtained straight from the reaction vial and were diffracted as such.

PBIA-Eu $\mathbf{u}_{\text {ACt }}$. White solid. FTIR ( $v \mathrm{~cm}^{-1}$, $\mathrm{KBr}$ pellet): 3600-2700 (O-H, $\left.\mathrm{H}_{2} \mathrm{O}_{\text {atm }}\right), 3100,3070,3039$, 3002, $\left(\mathrm{C}_{\mathrm{sp} 2}-\mathrm{H}\right), 2944\left(\mathrm{C}_{\mathrm{sp} 3}-\mathrm{H}\right), 1773,1717(-\mathrm{C}=\mathrm{O}-\mathrm{N}-\mathrm{C}=\mathrm{O}-), 1657 v_{\text {asym }}\left(\mathrm{COO}_{\text {mono }}\right), 1638\left(\mathrm{C}=\mathrm{O}_{\text {DMF }} \rightarrow \mathrm{Eu}\right)$, $1588 v_{\text {asym }}\left(\mathrm{COO}_{\text {iso }}\right), 1499,1465 \delta_{\text {asym }}\left(\mathrm{C}_{\mathrm{sp} 3}-\mathrm{H}\right), 1420 v_{\mathrm{sym}}(\mathrm{COO}), 1387\left(\mathrm{C}-\mathrm{N}_{\text {imide }}\right), 1367 \delta_{\text {sym }}\left(\mathrm{C}_{\mathrm{sp} 3}-\mathrm{H}\right), 1343$ $v_{\text {sym }}(\mathrm{COO}), 1285,1254,1169,1157,1132,1110,1083$ (C-O), 1024, 933, 874, 838, 803, 771, 731 (C-NC out of plane bending) 678, 628, 565, 405. PXRD ( ${ }^{\circ}, 2 \theta$ (count number)): 7.51 (110), 7.81 (328), 8.58 (790), 11.13 (112), 12.73 (156), 13.23 (90), 14.05 (199), 14.98 (106), 15.17 (82), 15.62 (229), $15 . .84$ (113), 17.12 (185), 17.49 (88), 18.47 (267), 19.10 (184), 19.39 (141), 19.93 (182), 20.91 (155), 21.27 (249), 22.05 (238), 22.44 (382), 23.03 8260), 23.39 (163), 25.79 (172), 26.45 (103), 27.45 (84), 28.18 (97), 30.75 (103), 31.90 (131), 32.30 (87), 33.40 (105), 33.65 (101), 35.76 (91) 37.15 (100) 37.62 (107). TGA (\% wt. loss, $\left.\mathrm{T}_{\text {interval }}\left[{ }^{\circ} \mathrm{C}\right]\right): 1,20-167 ; 9,167-354 ; 64,354-995$.

PBIA-Tb. Light green solid, 34.8\% yield. FTIR ( $v, \mathrm{~cm}^{-1}$, $\mathrm{KBr}$ pellet): 3600-2700 (O- $\left.\mathrm{H}, \mathrm{H}_{2} \mathrm{O}_{\mathrm{atm}}\right), 3100$, 3073, $3041\left(\mathrm{C}_{\mathrm{sp} 2}-\mathrm{H}\right), 2998,2945\left(\mathrm{C}_{\mathrm{sp} 3}-\mathrm{H}\right), 1773,1721(-\mathrm{C}=\mathrm{O}-\mathrm{N}-\mathrm{C}=\mathrm{O}-), 1660 v_{\text {asym }}\left(\mathrm{COO}_{\mathrm{mono}}\right), 1640$ $\left(\mathrm{C}=\mathrm{O}_{\mathrm{DMF}} \rightarrow \mathrm{Tb}\right), 1612 v_{\text {asym }}\left(\mathrm{COO}_{\text {aniso }}\right), 1590 v_{\text {asym }}\left(\mathrm{COO}_{\text {iso }}\right), 1498,1463 \delta_{\text {asym }}\left(\mathrm{C}_{\mathrm{sp} 3}-\mathrm{H}\right), 1421 v_{\text {sym }}(\mathrm{COO})$, 1386 (C-N imide $), 1361 \delta_{\text {sym }}\left(\mathrm{C}_{\text {sp3 }}-\mathrm{H}\right), 1346 v_{\text {sym }}(\mathrm{COO}), 1286,1253,1172,1158,1134,1108,1081$ (C-O), 1023, 931, 864, 838, 803, 783, 769, 733 (C-N-C out of plane bending), 679, 622, 564, 476, 412. PXRD

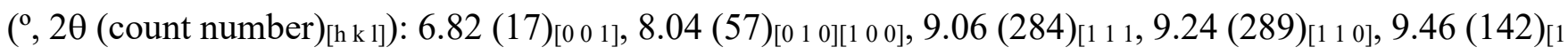

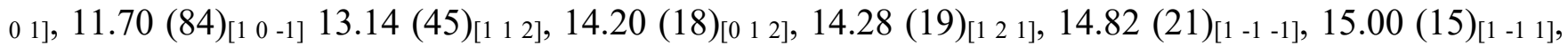

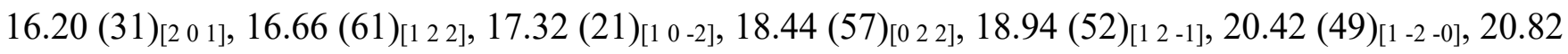

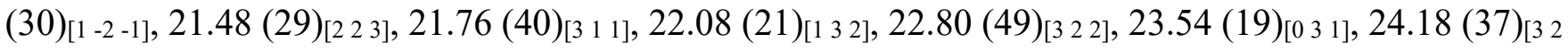

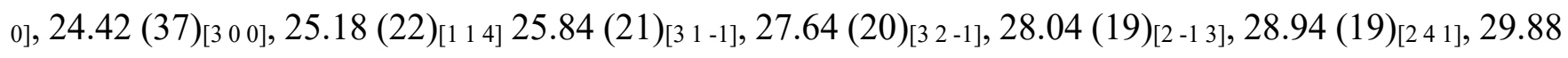


$\left.\left.\left.\left.(17)_{[2} 1-3\right], 30.44(19)_{[2} 43\right], 32.14(16)_{[4} 01\right], 32.4(28)_{[03} 34,36.62(18)_{[43}-1\right]$. TGA (\% wt. loss, $\left.T_{\text {interval }}\left[{ }^{\circ} \mathrm{C}\right]\right)$ : 4, 20-120; 6, 120-215; 8, 215-360; 56, 360-995. Besides, light green single crystals suitable for X-ray diffraction analysis were obtained straight from the reaction vial and were diffracted as such.

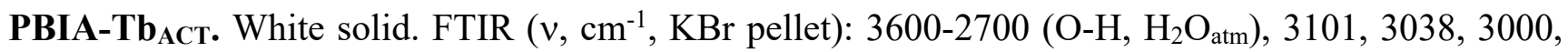
$\left(\mathrm{C}_{\mathrm{sp} 2}-\mathrm{H}\right), 2944\left(\mathrm{C}_{\mathrm{sp} 3}-\mathrm{H}\right), 1773,1717(-\mathrm{C}=\mathrm{O}-\mathrm{N}-\mathrm{C}=\mathrm{O}-), 1660 v_{\text {asym }}\left(\mathrm{COO}_{\mathrm{mono}}\right), 1637\left(\mathrm{C}=\mathrm{O}_{\text {DMF }} \rightarrow \mathrm{Tb}\right), 1590$ $v_{\text {asym }}\left(\mathrm{COO}_{\text {iso }}\right), 1501,1464 \delta_{\text {asym }}\left(\mathrm{C}_{\text {sp } 3-H}\right), 1421 v_{\text {sym }}(\mathrm{COO}), 1388\left(\mathrm{C}-\mathrm{N}_{\text {imide }}\right), 1366 \delta_{\text {sym }}\left(\mathrm{C}_{\text {sp } 3}-\mathrm{H}\right), 1343$ $v_{\text {sym }}(\mathrm{COO}), 1285,1170,1157,1134,1109,1083$ (C-O), 1024, 934, 875, 839, 802, 771, 731 (C-N-C out of plane bending) 679, 629, 564, 408. PXRD ( ${ }^{\circ}, 2 \theta$ (count number)): 7.46 (349), 7.79 (564), 8.51 (1403), 9.25 (115), 9.92 (78), 11.19 (303), 11.64 (104), 12.67 (214), 12.84 (206), 13.33 (127), 14.02 (309), 14.98 (237), 15.24 (113), 15.62 (285), 17.04 (201), 17.50 (135), 17.89 (88), 18.49 (285), 19.19 (257), 19.39 (208), 19.81 (165), 20.04 (178), 20.88 (211), 21.29 (393), 22.09 (274), 22.53 (672), 23.17 (399), 23.44 (306), 24.07 (125), 25.47 (237), 26.41 (115), 27.53 (97), 28.19 (141), 28.89 (76), 29.37 (90), 31.87 (156), 33.65 (121), 34.63 (115), 37.79 (139), 43.17 (101), 46.82 (98), 48.15 (107). TGA (\% wt. loss, Tinterval $\left.\left[{ }^{\circ} \mathrm{C}\right]\right): 2,20-165 ; 8,165-365 ; 65,365-995$.

PBIA-Er. Light pink solid, 36\% yield FTIR ( $v, \mathrm{~cm}^{-1}, \mathrm{KBr}$ pellet): 3600-2700 (O-H, $\mathrm{H}_{2} \mathrm{O}_{\mathrm{atm}}$ ), 3100, 3075, $3043\left(\mathrm{C}_{\text {sp2 }-\mathrm{H}}\right), 2997,2945\left(\mathrm{C}_{\mathrm{sp} 3}-\mathrm{H}\right), 1774,1721$ (-C=O-N-C=O-), 1663 vasym $\left(\mathrm{COO}_{\text {mono }}\right), 1643$ $\left(\mathrm{C}=\mathrm{O}_{\mathrm{DMF}} \rightarrow \mathrm{Er}\right), 1605 v_{\text {asym }}\left(\mathrm{COO}_{\text {aniso-iso }}\right), 1499,1465 \delta_{\text {asym }}\left(\mathrm{C}_{\mathrm{sp} 3}-\mathrm{H}\right), 1424 v_{\mathrm{sym}}(\mathrm{COO}), 1386\left(\mathrm{C}-\mathrm{N}_{\text {imide }}\right), 1362$ $\delta_{\text {sym }}\left(\mathrm{C}_{\text {sp } 3}-\mathrm{H}\right), 1349 v_{\text {sym }}(\mathrm{COO}), 1286,1253,1173,1158,1134,1108,1081$ (C-O), 1023, 933, 866, 838, 803, 782, 769, 732 (C-N-C out of plane bending), 682, 629, 564, 479, 415. PXRD ( ${ }^{\circ}, 2 \theta$ (count number) $)_{[\mathrm{h}}$

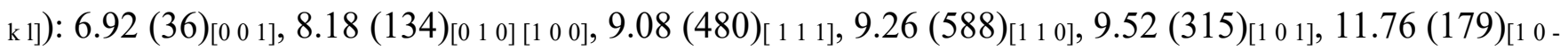

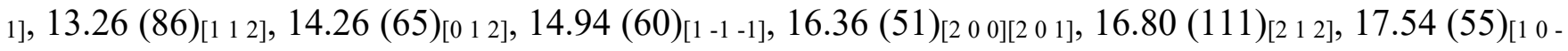

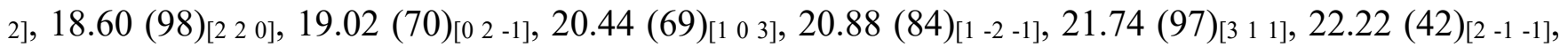




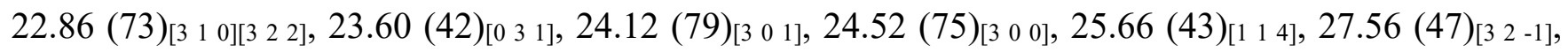

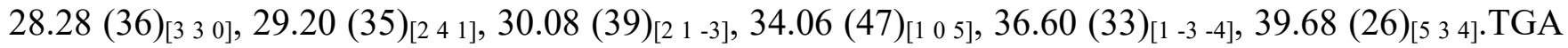
(\% wt. loss, $\left.\mathrm{T}_{\text {interval }}\left[{ }^{\circ} \mathrm{C}\right]\right): 3,20-115 ; 9,115-235 ; 5,235-365 ; 59,365-995$. Besides, light pink single crystals suitable for X-ray diffraction analysis were obtained straight from the reaction vial and were diffracted as such.

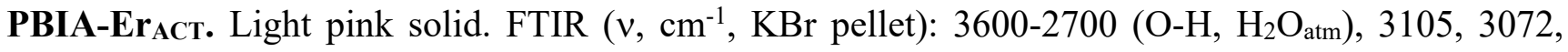
3045, 3000, $\left(\mathrm{C}_{\mathrm{sp} 2}-\mathrm{H}\right), 2944\left(\mathrm{C}_{\mathrm{sp} 3}-\mathrm{H}\right), \quad 1774,1718 \quad(-\mathrm{C}=\mathrm{O}-\mathrm{N}-\mathrm{C}=\mathrm{O}-), 1660 \quad v_{\text {asym }}\left(\mathrm{COO}_{\text {mono }}\right), 1640$ $\left(\mathrm{C}=\mathrm{O}_{\mathrm{DMF}} \rightarrow \mathrm{Er}\right), 1598 v_{\text {asym }}(\mathrm{COO}), 1498,1464 \delta_{\text {asym }}\left(\mathrm{C}_{\mathrm{sp} 3}-\mathrm{H}\right), 1425 v_{\text {sym }}(\mathrm{COO}), 1388\left(\mathrm{C}-\mathrm{N}_{\text {imide }}\right), 1366$ $\delta_{\mathrm{sym}}\left(\mathrm{C}_{\mathrm{sp} 3}-\mathrm{H}\right), 1345 v_{\mathrm{sym}}(\mathrm{COO}), 1285,1254,1171,1157,1133,1083,(\mathrm{C}-\mathrm{O}), 1023,935,874,839,803,770$, 731 (C-N-C out of plane bending) 684, 630, 565, 406. PXRD ( ${ }^{\circ}, 2 \theta$ (count number)): 8.05 (756), 8.37 (947), 8.97 (1115), 9.88 (414), 12.53 (412), 13.04 (514), 13.62 (236), 14.39 (504), 15.36 (210), 16.69 (309), 17.29 (319), 18.55 (334), 19.34 (401), 20.81 (415), 21.92 (404), 22.34 (341), 23.44 (361), 25.70 (298). TGA (\% wt. loss, $\left.\mathrm{T}_{\text {interval }}\left[{ }^{\circ} \mathrm{C}\right]\right): 3,20-187 ; 4,187-345 ; 67,345-995$.

PBIA-Tm. Light green solid, 31.8\% yield. FTIR ( $v, \mathrm{~cm}^{-1}$, KBr pellet): 3600-2700 (O-H, $\left.\mathrm{H}_{2} \mathrm{O}_{\mathrm{atm}}\right), 3102$, 3075, $3043\left(\mathrm{C}_{\mathrm{sp} 2}-\mathrm{H}\right), 2997,2946\left(\mathrm{C}_{\mathrm{sp} 3}-\mathrm{H}\right), 1774,1721$ (-C=O-N-C=O-), 1663 vasym $\left(\mathrm{COO}_{\text {mono }}\right), 1643$ $\left(\mathrm{C}=\mathrm{O}_{\mathrm{DMF}} \rightarrow \mathrm{Tm}\right), 1603 v_{\text {asym }}\left(\mathrm{COO}_{\text {aniso-iso }}\right), 1498,1465 \delta_{\text {asym }}\left(\mathrm{C}_{\mathrm{sp} 3}-\mathrm{H}\right), 1425 v_{\mathrm{sym}}(\mathrm{COO}), 1386\left(\mathrm{C}-\mathrm{N}_{\text {imide }}\right)$, $1362 \delta_{\text {sym }}\left(\mathrm{C}_{\mathrm{sp} 3}-\mathrm{H}\right), 1351 v_{\text {sym }}(\mathrm{COO}), 1286,1253,1173,1158,1134,1108,1081$ (C-O), 1023, 933, 866, 838, 804, 784, 769, 732 (C-N-C out of plane bending), 682, 628, 564, 483, 411. PXRD ( ${ }^{\circ}, 2 \theta$ (count

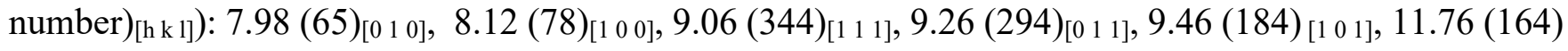

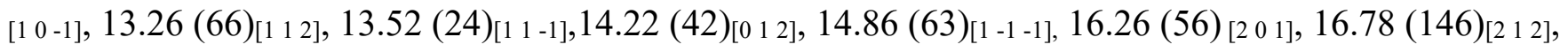

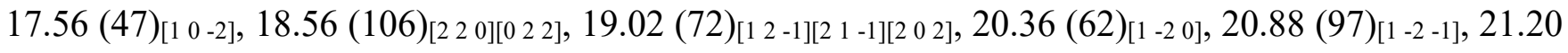

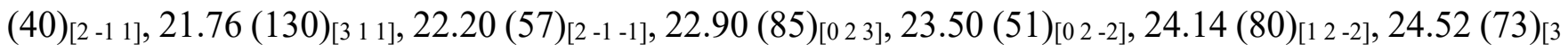




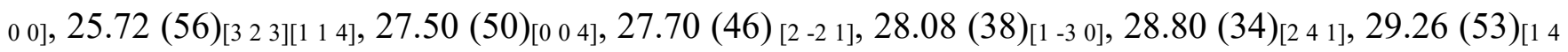

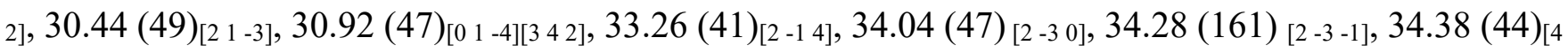
$\left.\left.2-1], 35.32(33)_{[4} 03\right], 36.66(34)_{[2} 1-4\right], 38.84(36)_{[2-3-3]}, 40.48(32)_{[4} 51$ 1]. TGA (\% wt. loss, $\left.T_{\text {interval }}\left[{ }^{\circ} \mathrm{C}\right]\right): 2$, 20-68; 4, 68-155; 9, 155-265; 3, 265-334; 59, 334-995. Besides, light green single crystals suitable for Xray diffraction analysis were obtained straight from the reaction vial and were diffracted as such.

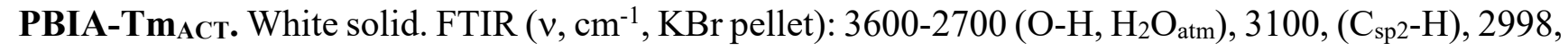
$2944\left(\mathrm{C}_{\text {sp } 3}-\mathrm{H}\right), \quad 1774,1719 \quad(-\mathrm{C}=\mathrm{O}-\mathrm{N}-\mathrm{C}=\mathrm{O}-), 1663 \quad v_{\text {asym }}\left(\mathrm{COO}_{\text {mono }}\right), 1644 \quad\left(\mathrm{C}=\mathrm{O}_{\text {DMF }} \rightarrow \mathrm{Tm}\right), 1603$ $v_{\text {asym }}(\mathrm{COO}), 1497,1464 \delta_{\text {asym }}\left(\mathrm{C}_{\text {sp3 } 3}-\mathrm{H}\right), 1427 v_{\text {sym }}(\mathrm{COO}), 1388\left(\mathrm{C}-\mathrm{N}_{\text {imide }}\right), 1366 \delta_{\text {sym }}\left(\mathrm{C}_{\mathrm{sp} 3}-\mathrm{H}\right), 1345$ $v_{\text {sym }}(\mathrm{COO}), 1285,1252,1172,1157,1133,1082,(\mathrm{C}-\mathrm{O}), 1023,935,875,839,803,769,731$ (C-N-C out of plane bending) 684, 629, 564, 404. PXRD ( ${ }^{\circ}, 2 \theta$ (count number)): 8.07 (570), 8.36 (662), 8.92 (774), 11.25 (225), 12.60 (405), 13.10 (387), 14.29 (435), 15.37 (246), 16.72 (255), 17.35 (294), 19.48 (355), 20.66 (350), 21.35 (360), 21.87 (361), 23.26 (322), 31.23 (220). TGA (\% wt. loss, Tinterval $\left.\left[{ }^{\circ} \mathrm{C}\right]\right): 3,20-$ 168; 4, 168-336; 62, 336-995. 


\section{NMR of the PBIA ligand}

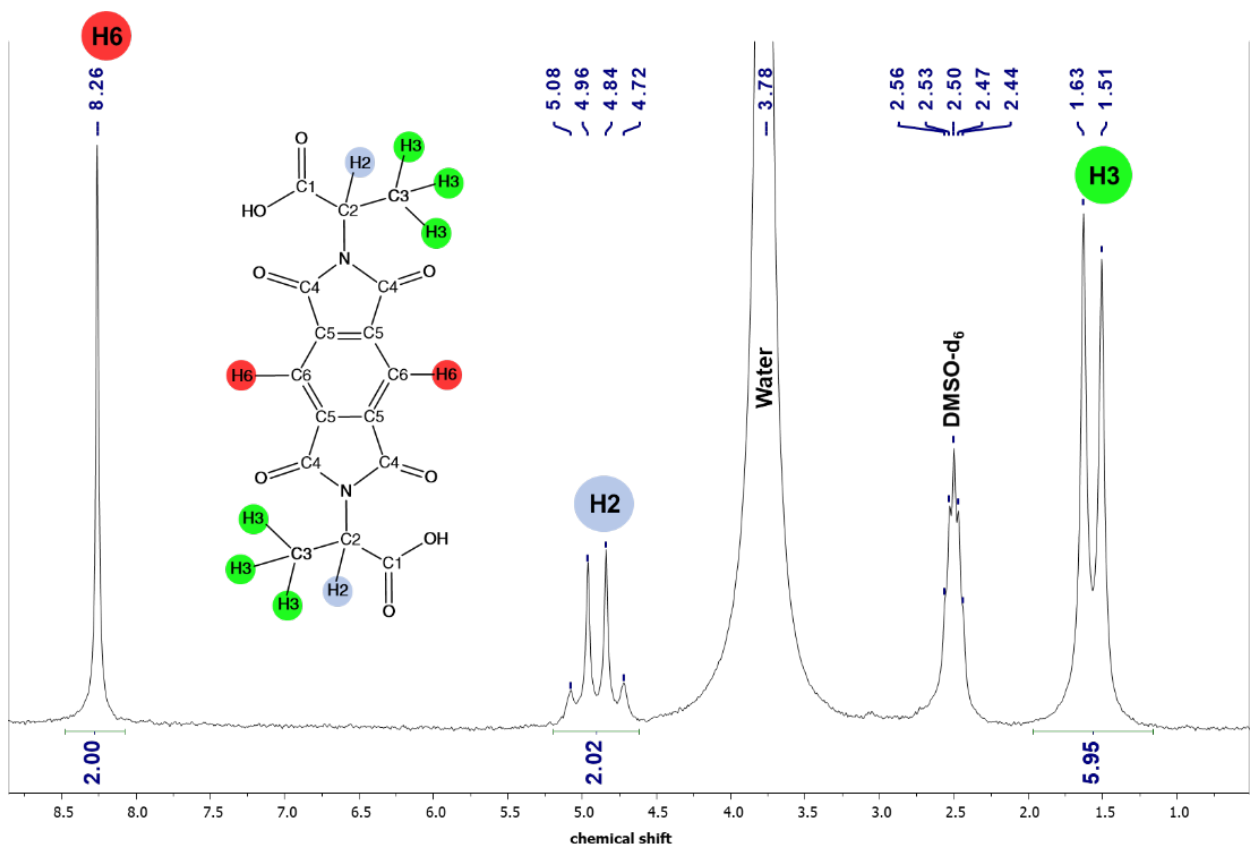

Figure S1. ${ }^{1} \mathrm{H}$ NMR spectrum of PBIA ligand at $60 \mathrm{MHz}\left(\mathrm{DMSO}-d_{6} \& \mathrm{D}_{2} \mathrm{O}\right)$.

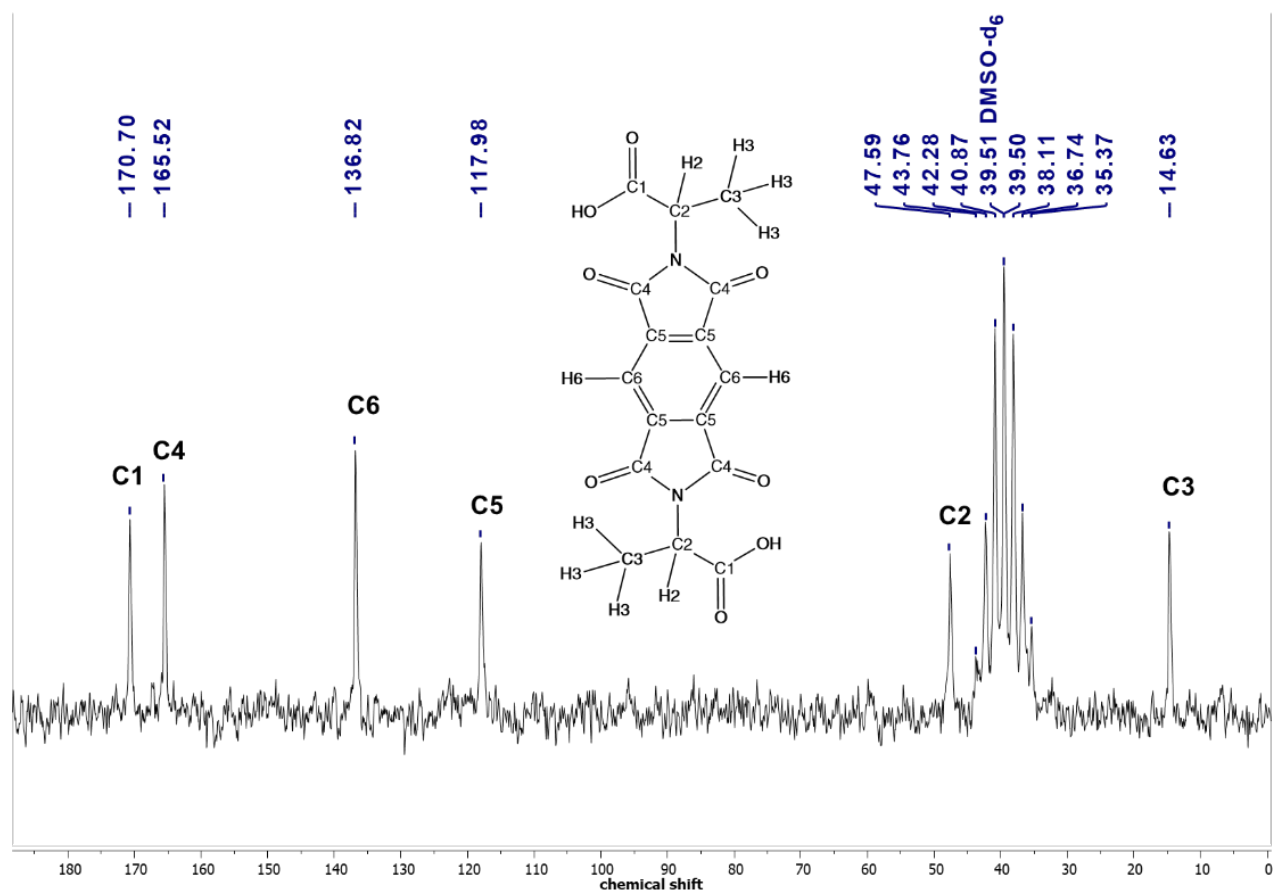

Figure S2. ${ }^{13} \mathrm{C}$ NMR spectrum of PBIA ligand at $15 \mathrm{MHz}\left(\mathrm{DMSO}-d_{6} \& \mathrm{D}_{2} \mathrm{O}\right)$. 


\section{FTIR}

Comparison between inactivated and activated materials.
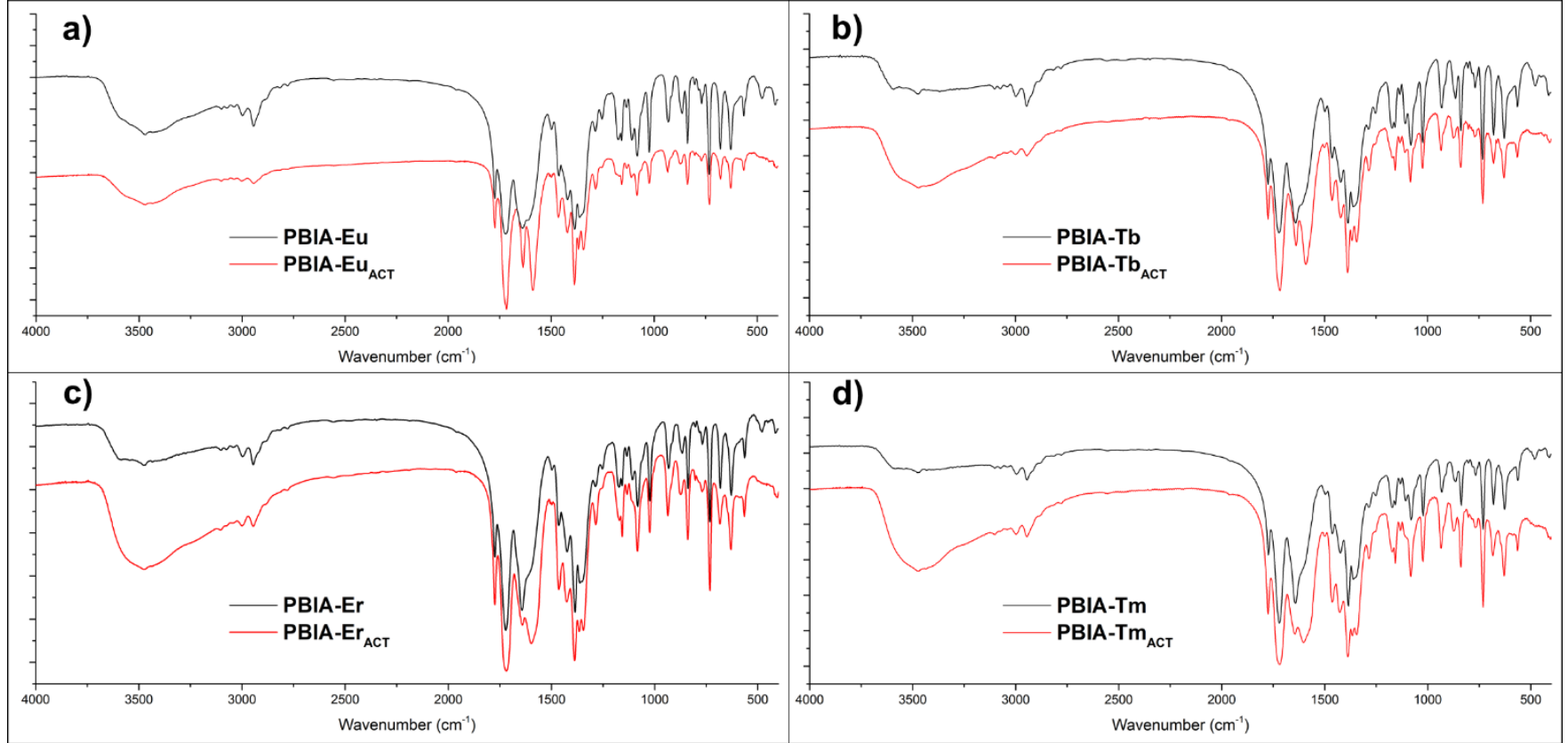

Figure S3. Comparison between a) PBIA-Eu and PBIA-EuACT; b) PBIA-Tb and PBIA-TbACT; c) PBIA-Er and PBIA-ErACT; d) PBIA-Tm and PBIA-TmACT. 


\section{Single crystal X-ray selected geometrical parameters}

\begin{tabular}{|c|c|c|c|c|c|}
\hline \multicolumn{6}{|c|}{$\begin{array}{l}\text { Table S1. Single crystal X-ray selected geometrical parameters of compounds PBIA-Pr, PBIA-Eu, PBIA-Tb, PBIA-Er an } \\
\text { PBIA-Tm obtained by X-ray crystal analysis. }\end{array}$} \\
\hline Distances $[\AA]$ & PBIA-Pr & PBIA-Eu & PBIA-Tb & PBIA-Er & PBIA-Tm \\
\hline $\mathrm{M}_{\mathrm{Ln}-\mathrm{O}(1)_{\text {ESTER-SYN }}}$ & $2.3456(19)$ & $2.282(2)$ & $2.234(13)$ & $2.221(4)$ & $2.214(4)$ \\
\hline $\mathrm{M}_{\mathrm{Ln}}-\mathrm{O}(1 \mathrm{~A})_{\text {ESTER-SYN }}$ & - & 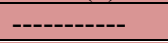 & $2.281(10)$ & - & 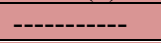 \\
\hline $\mathrm{M}_{\mathrm{Ln}-} \mathrm{O}(19)_{\text {IIO-SYN }}$ & $2.4575(19)$ & $2.372(2)$ & $2.342(3)$ & $2.300(4)$ & $2.292(4)$ \\
\hline $\mathrm{M}_{\mathrm{Ln}}-\mathrm{O}(18)_{\text {ISO-SYN }}$ & $2.464(2)$ & $2.379(2)$ & $2.347(3)$ & $2.304(4)$ & $2.297(4)$ \\
\hline $\mathrm{M}_{\mathrm{Ln}} \mathrm{O}(27)_{\mathrm{ANISO}-\mathrm{T}}$ & $2.955(2)$ & $3.066(3)$ & $3.1458(2)$ & $3.181(5)$ & $3.2092(3)$ \\
\hline $\mathrm{M}_{\mathrm{Ln}-} \mathrm{O}(39)_{\mathrm{ANISO}-\mathrm{ANTI}}$ & $2.4944(19)$ & $2.399(2)$ & $2.358(3)$ & $2.315(4)$ & $2.306(4)$ \\
\hline $\mathrm{M}_{\mathrm{Ln}}-\mathrm{O}(27)_{\text {ANISO-ANTI }}$ & $2.4518(18)$ & $2.349(2)$ & $2.306(3)$ & $2.261(4)$ & $2.252(2)$ \\
\hline $\mathrm{M}_{\mathrm{Ln}}-\mathrm{O}(45)_{\mathrm{DMF} 1}$ & $2.513(2)$ & $2.435(2)$ & $2.401(3)$ & $2.358(5)$ & $2.356(4)$ \\
\hline $\mathrm{M}_{\mathrm{Ln}}-\mathrm{O}(40)_{\mathrm{DMF} 2}$ & $2.469(2)$ & $2.436(16)$ & $2.415(14)$ & $2.36(2)$ & $2.343(10)$ \\
\hline $\mathrm{M}_{\mathrm{Ln}}-\mathrm{O}(40 \mathrm{~A})_{\mathrm{DMF} 2 \mathrm{~A}}$ & --o------- & $2.380(16)$ & $2.349(13)$ & $2.331(19)$ & $2.335(10)$ \\
\hline $\mathrm{M}_{\mathrm{Ln}}-\mathrm{O}(50)_{\mathrm{DMF} 3}$ & $2.527(2)$ & $2.490(4)$ & $2.453(6)$ & $2.445(10)$ & $2.445(9)$ \\
\hline $\mathrm{M}_{\mathrm{Ln}}-\mathrm{M}_{\mathrm{Ln}[\mathrm{CN}=9]}$ & $4.2707(3)$ & $4.2522(4)$ & $4.2565(3)$ & $4.2380(6)$ & $4.2365(3)$ \\
\hline $\mathrm{O}(27)-\mathrm{O}(27)_{[\mathrm{CN}=9]}$ & $3.3538(2)$ & $3.428(5)$ & $3.5083(2)$ & $3.536(10)$ & $3.5766(3)$ \\
\hline $\mathrm{O}(1)-\mathrm{C}(2)_{\text {ESTER-SYN }}$ & $1.266(3)$ & $1.266(4)$ & $1.203(13)$ & $1.260(7)$ & $1.260(6)$ \\
\hline $\mathrm{O}(26)-\mathrm{C}(2)_{\text {ESTER-SYN }}$ & $1.222(3)$ & $1.224(4)$ & $1.235(10)$ & $1.223(7)$ & $1.228(7)$ \\
\hline $\mathrm{O}(27)-\mathrm{C}(28)_{\text {ANISO-ANTI }}$ & $1.256(3)$ & $1.250(4)$ & $1.247(4)$ & $1.249(7)$ & $1.246(6)$ \\
\hline $\mathrm{O}(39)-\mathrm{C}(28)_{\text {ANISO-ANTI }}$ & $1.234(3)$ & $1.242(4)$ & $1.237(5)$ & $1.235(7)$ & $1.240(7)$ \\
\hline $\mathrm{O}(18)-\mathrm{C}(17)_{\text {ISO-SYN }}$ & $1.247(3)$ & $1.241(4)$ & $1.239(4)$ & $1.238(7)$ & $1.246(6)$ \\
\hline $\mathrm{O}(19)-\mathrm{C}(17)_{\mathrm{ISO}-\mathrm{SYN}}$ & $1.251(3)$ & $1.252(4)$ & $1.249(4)$ & $1.256(7)$ & $1.257(6)$ \\
\hline $\mathrm{O}(45)-\mathrm{C}(46)_{\mathrm{DMF} 1}$ & $1.213(5)$ & $1.231(5)$ & $1.219(7)$ & $1.219(8)$ & $1.235(7)$ \\
\hline $\mathrm{O}(40)-\mathrm{C}(41)_{\mathrm{DMF} 2}$ & $1.226(4)$ & $1.23(2)$ & $1.139(17)$ & $1.20(3)$ & $1.21(2)$ \\
\hline $\mathrm{O}(40 \mathrm{~A})-\mathrm{C}(41 \mathrm{~A})_{\mathrm{DMF} 2 \mathrm{~A}}$ & - & 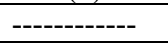 & $1.27(2)$ & $1.24(2)$ & $1.24(2)$ \\
\hline $\mathrm{O}(50)-\mathrm{C}(51)_{\mathrm{DMF} 3}$ & $1.221(4)$ & $1.199(10)$ & $1.17(3)$ & $1.11(4)$ & $1.15(3)$ \\
\hline $\mathrm{O}(50)-\mathrm{C}(51 \mathrm{~A})_{\mathrm{DMF} 3 \mathrm{~A}}$ & - n & $1.209(19)$ & $1.20(2)$ & $1.21(3)$ & $1.24(3)$ \\
\hline \multicolumn{6}{|l|}{ Bond angles $\left[{ }^{\circ}\right]$} \\
\hline $\mathrm{O}(27)-\mathrm{M}_{\mathrm{Ln}}-\mathrm{O}(39)_{[\mathrm{CN}=9]}$ & $122.73(7)$ & $122.96(9)$ & $122.82(12)$ & $123.09(17)$ & $123.22(16)$ \\
\hline $\mathrm{O}(27)-\mathrm{M}_{\mathrm{Ln}}-\mathrm{O}(27)_{[\mathrm{CN}=9]}$ & $76.04(7)$ & $77.30(8)$ & $78.449(3)$ & $76.06(16)$ & $9.725(3)$ \\
\hline $\mathrm{M}_{\mathrm{Ln}}-\mathrm{O}(27)-\mathrm{M}_{\mathrm{Ln}[\mathrm{CN}=9]}$ & $103.96(7)$ & $102.69(8)$ & $101.551(3)$ & $100.94(16)$ & $100.275(3)$ \\
\hline $\mathrm{O}(1)_{\text {ESTER }}-\mathrm{M}_{\mathrm{Ln}}-\mathrm{O}(39)_{\text {ANISO-ANTI }}$ & $80.62(7)$ & $83.14(9)$ & $81.3(4)$ & $85.79(17)$ & $86.46(16)$ \\
\hline $\mathrm{O}(1)_{\text {ESTER-SYN }}-\mathrm{M}_{\mathrm{Ln}}-\mathrm{O}(19)_{\text {IISO-SYN }}$ & $140.34(7)$ & $143.42(9)$ & $139.6(3)$ & $145.83(17)$ & $146.57(15)$ \\
\hline O(45)DMF1-M ${ }_{\mathrm{Ln}-} \mathrm{O}(40) \mathrm{DMF} 2$ & $68.53(10)$ & $68.1(3)$ & $78.4(3)$ & $68.1(4)$ & $68.9(4)$ \\
\hline $\mathrm{O}(45)_{\mathrm{DMF} 1}-\mathrm{M}_{\mathrm{Ln}}-\mathrm{O}(40 \mathrm{~A})_{\mathrm{DMF} 2 \mathrm{~A}}$ & 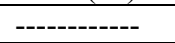 & 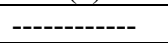 & $66.5(3)$ & $79.0(4)$ & $79.6(4)$ \\
\hline $\mathrm{O}(40)_{D_{\text {MF } 2}}-\mathrm{M}_{\mathrm{Ln}}-\mathrm{O}(50)_{\mathrm{DMF} 3}$ & $70.91(8)$ & $71.2(3)$ & $72.1(3)$ & $71.2(5)$ & $70.3(4)$ \\
\hline $\mathrm{O}(40 \mathrm{~A})_{\mathrm{DMF} 2 \mathrm{~A}}-\mathrm{M}_{\mathrm{Ln}} \mathrm{O}(50)_{\mathrm{DMF} 3}$ & 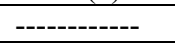 & 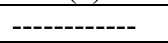 & $59.1(3)$ & $57.8(4)$ & $56.7(4)$ \\
\hline $\mathrm{M}_{\mathrm{Ln}} \mathrm{O}(27)-\mathrm{C}(28)_{\text {ANISO-ANTI }}$ & $82.90(17)$ & $78.3(2)$ & $75.593(3)$ & $73.3(4)$ & $72.864(3)$ \\
\hline $\mathrm{M}_{\mathrm{Ln}}-\mathrm{O}(39)-\mathrm{C}(28)_{\text {ANISO-ANTI }}$ & $105.54(16)$ & $110.69(19)$ & $114.3(2)$ & $116.1(4)$ & $117.3(3)$ \\
\hline $\mathrm{M}_{\mathrm{Ln}} \mathrm{O}(27)-\mathrm{C}(28)_{\text {ANISO-T }}$ & $171.0(2)$ & $174.4(2)$ & $172.7(3)$ & $171.5(5)$ & $169.766(1)$ \\
\hline $\mathrm{M}_{\mathrm{Ln}-} \mathrm{O}(1)-\mathrm{C}(2)_{\text {ESTER-SYN }}$ & $148.1(2)$ & $148.0(2)$ & $161.132(1)$ & $147.7(5)$ & $146.9(4)$ \\
\hline $\mathrm{M}_{\mathrm{Ln}}-\mathrm{O}(1 \mathrm{~A})-\mathrm{C}(2)_{\text {ESTER-SYN }}$ & 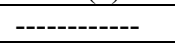 & 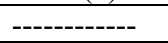 & $138.608(2)$ & 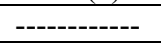 & 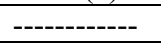 \\
\hline $\mathrm{M}_{\mathrm{Ln}-} \mathrm{O}(18)-\mathrm{C}(17)_{\text {ISO-SYN }}$ & $136.86(19)$ & $138.2(2)$ & $138.392(2)$ & $139.0(4)$ & $138.7(4)$ \\
\hline $\mathrm{M}_{\mathrm{Ln}}-\mathrm{O}(19)-\mathrm{C}(17)_{\mathrm{ISO}-\mathrm{SYN}}$ & $139.09(18)$ & $139.5(2)$ & 140.4(3) & $140.6(4)$ & 141.1(4) \\
\hline \multicolumn{6}{|l|}{ Dihedral angles $\left[{ }^{\circ}\right]$} \\
\hline $\mathrm{M}_{\mathrm{Ln}}-\mathrm{O}(1)-\mathrm{C}(2)-\mathrm{O}(26)_{\text {ESTER-SYN }}$ & $74.661(6)$ & $67.7(6)$ & $-56.004(4)$ & $-63.8(13)$ & $-63.6(12)$ \\
\hline $\mathrm{M}_{\mathrm{Ln}}-\mathrm{O}(1 \mathrm{~A})-\mathrm{C}(2)-\mathrm{O}(26 \mathrm{~A})_{\text {ESTER-SYN }}$ & 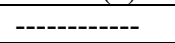 & 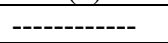 & $-60.995(5)$ & 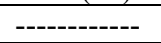 & 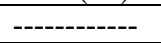 \\
\hline $\mathrm{M}_{\mathrm{Ln}}-\mathrm{O}(19)-\mathrm{C}(17)-\mathrm{O}(18)_{\text {ISO-SYN }}$ & $32.994(3)$ & $33.4(6)$ & $-32.832(2)$ & $-31.8(11)$ & $-30.9(11)$ \\
\hline $\mathrm{M}_{\mathrm{Ln}}-\mathrm{O}(18)-\mathrm{C}(17)-\mathrm{O}(19)_{\mathrm{ISO}-\mathrm{SYN}}$ & $1.0048(1)$ & $-1.1(6)$ & $3.1008(3)$ & $1.2(12)$ & $1.9(11)$ \\
\hline $\mathrm{M}_{\mathrm{Ln}}-\mathrm{O}(39)-\mathrm{C}(28)-\mathrm{O}(27)_{\text {ANISO-ANTI }}$ & $-8.7788(5)$ & $-8.4(4)$ & $-8.808(4)$ & $8.6(9)$ & $8.9(8)$ \\
\hline $\mathrm{M}_{\mathrm{Ln}}-\mathrm{O}(19)-\mathrm{O}(1)-\mathrm{M}_{\mathrm{Ln}}$ SBU-ISO-SBU & $-10.3043(7)$ & $-20.17(9)$ & $-156.019(2)$ & $19.9(3)$ & $161.519(1)$ \\
\hline $\mathrm{O}(1)-\mathrm{M}_{\mathrm{Ln}}-\mathrm{M}_{\mathrm{Ln}} \mathrm{O}(1)$ ISO-SBU-ISO & $-180.00(5)$ & $-180.0(2)$ & $-180.00(5)$ & $-180.0(2)$ & $180.00(4)$ \\
\hline $\mathrm{M}_{\mathrm{Ln}-} \mathrm{O}(27)-\mathrm{O}(27)-\mathrm{M}_{\mathrm{Ln}}$ SBU-ANTI-SBU & $-180.00(4)$ & $-180.00(3)$ & $180.00(4)$ & $-180.00(4)$ & $-180.00(8)$ \\
\hline $\mathrm{M}_{\mathrm{Ln}}-\mathrm{C}(28)-\mathrm{O}(27)-\mathrm{M}_{\mathrm{Ln}}$ & -39.126 & $-177.34(11)$ & $176.726(3)$ & $-176.9(2)$ & $-47.958(5)$ \\
\hline
\end{tabular}




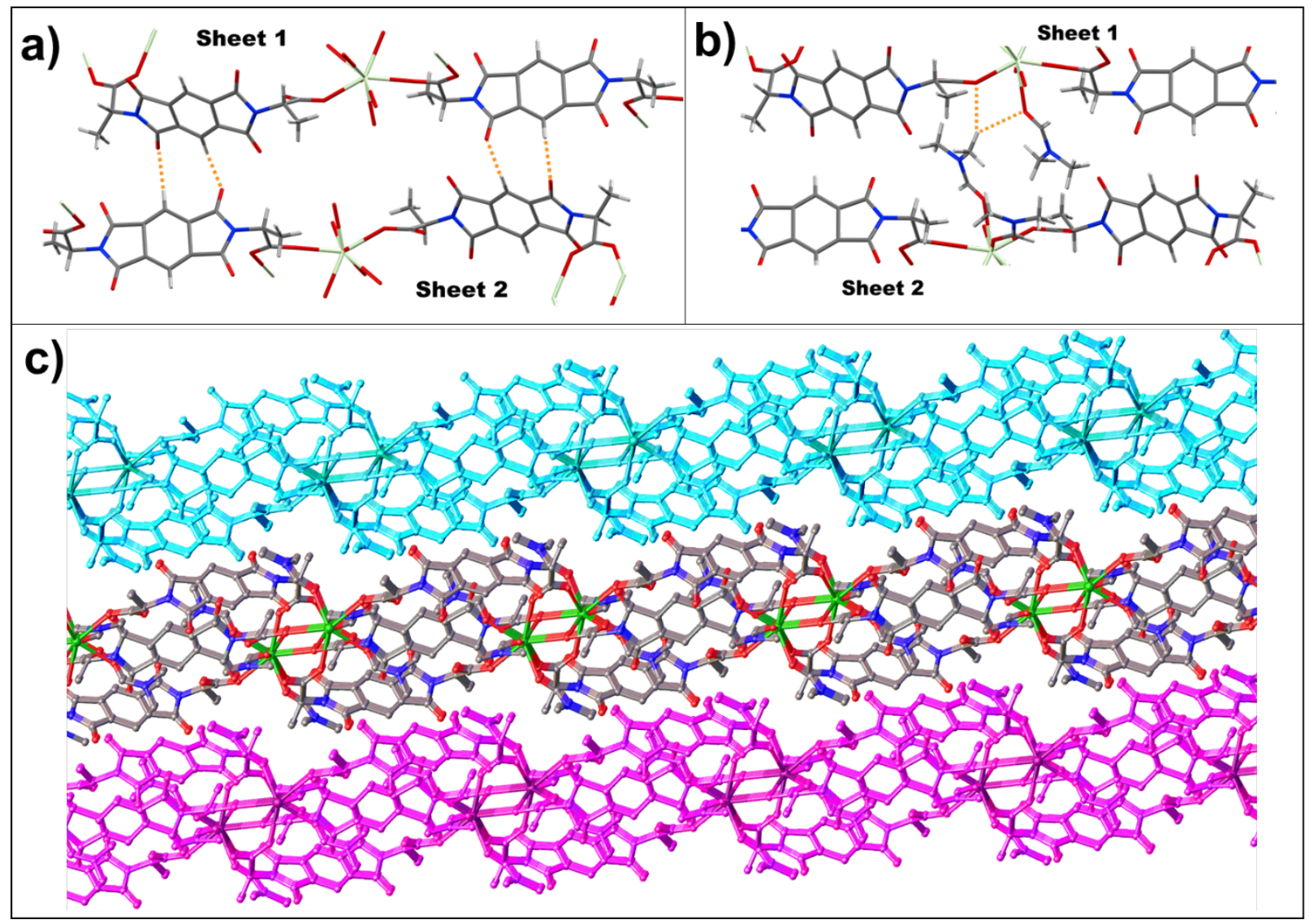

Figure S4. a) and b) show hydrogen bonding interactions between fragments from different sheets. c) Side-view of three stacked 2D sheets. 
ORTEP plots of PBIA-Ln single crystal structures (asymmetric unit)

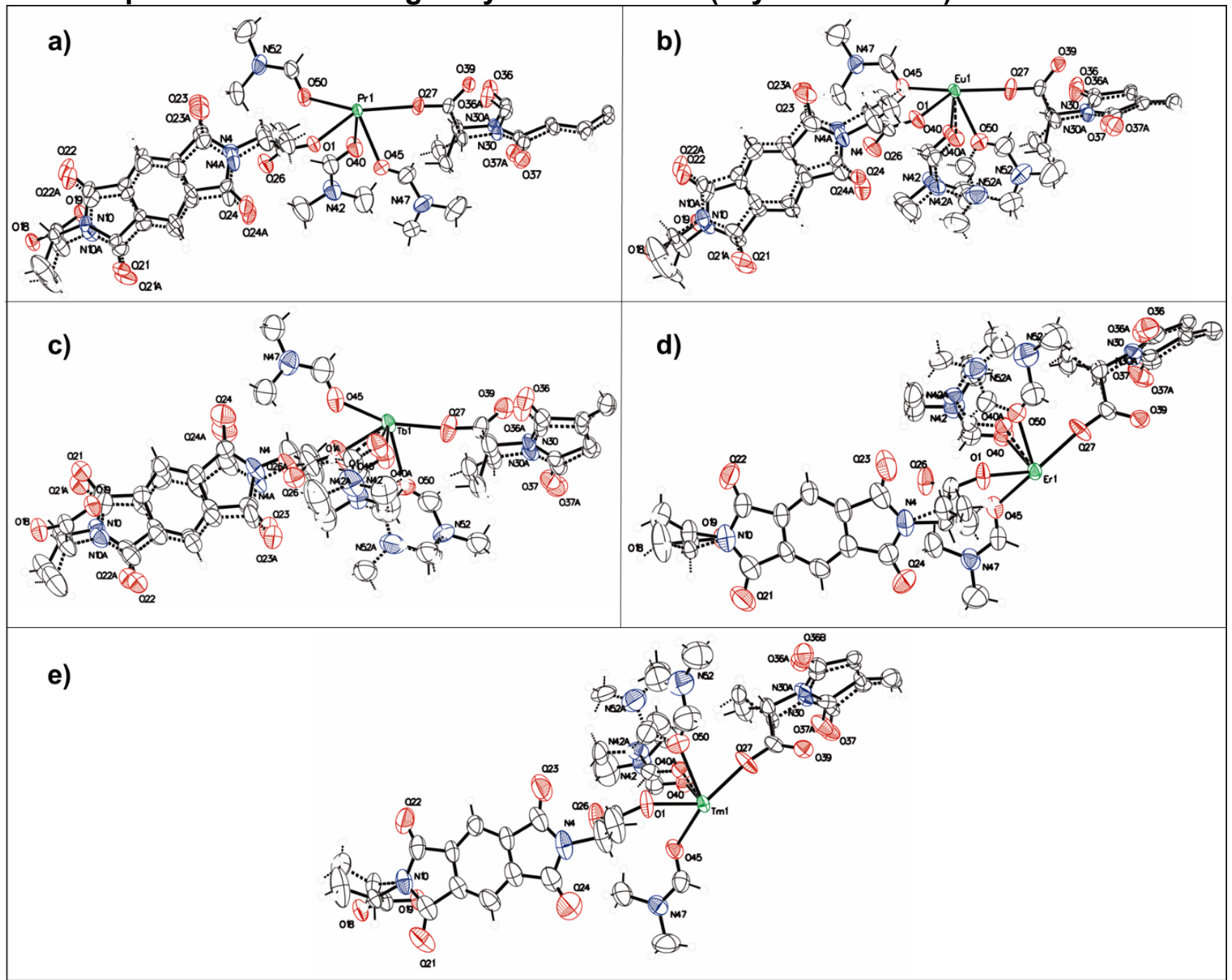

Figure S5. ORTEP plots of a) PBIA-Pr; b) PBIA-Eu; c) PBIA-Tb; d) PBIA-Er; e) PBIA-Tm. 


\section{PXRD}

Comparison between simulated (from single crystal structure) and experimental powder X-ray diffraction patterns.

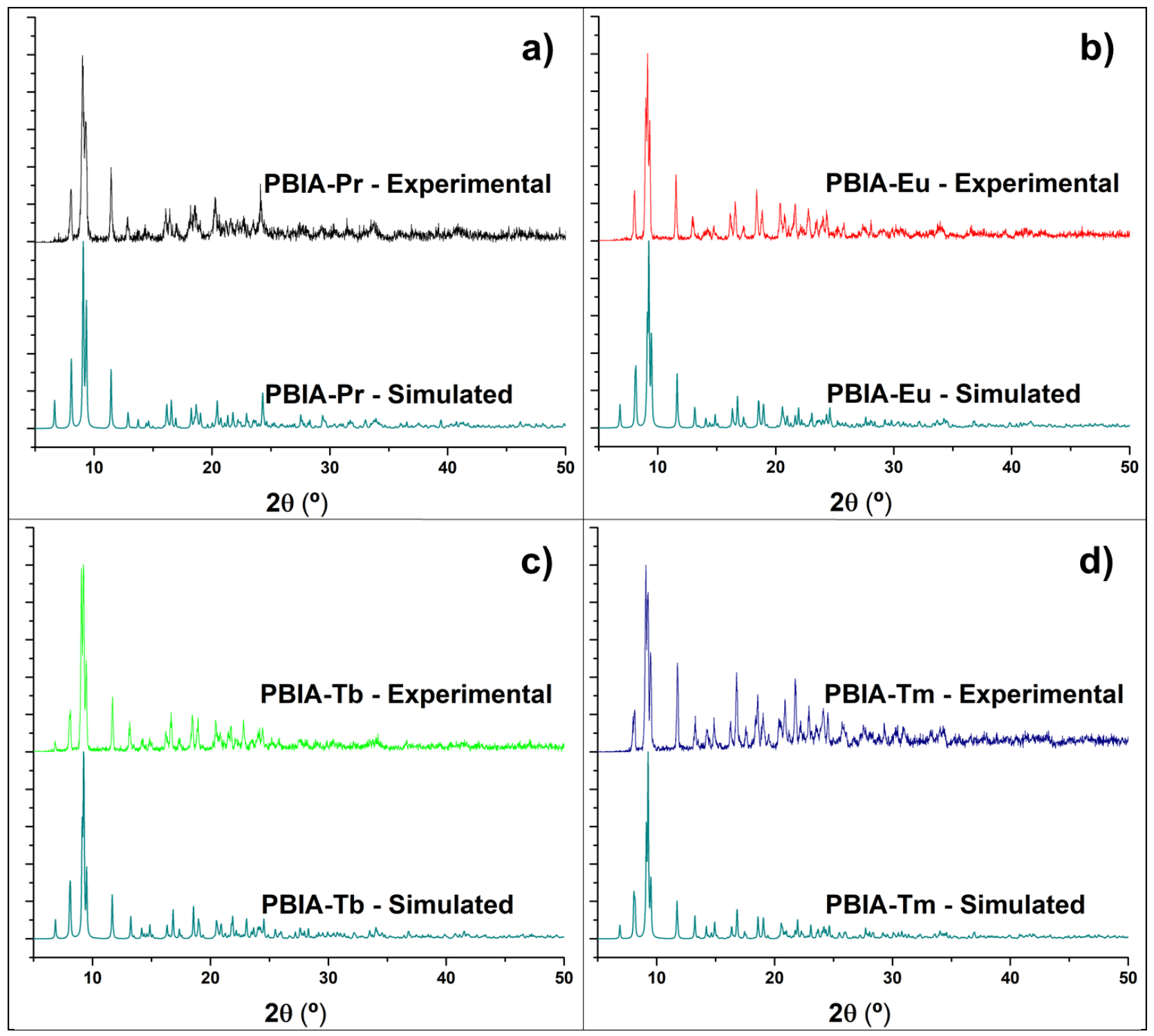

Figure S6. Comparison between experimental and simulated X-ray diffractograms of a) PBIAPr; b) PBIA-Eu; c) PBIA-Tb; d) PBIA-Tm. 
Comparison between X-ray diffractograms of inactivated and activated materials.

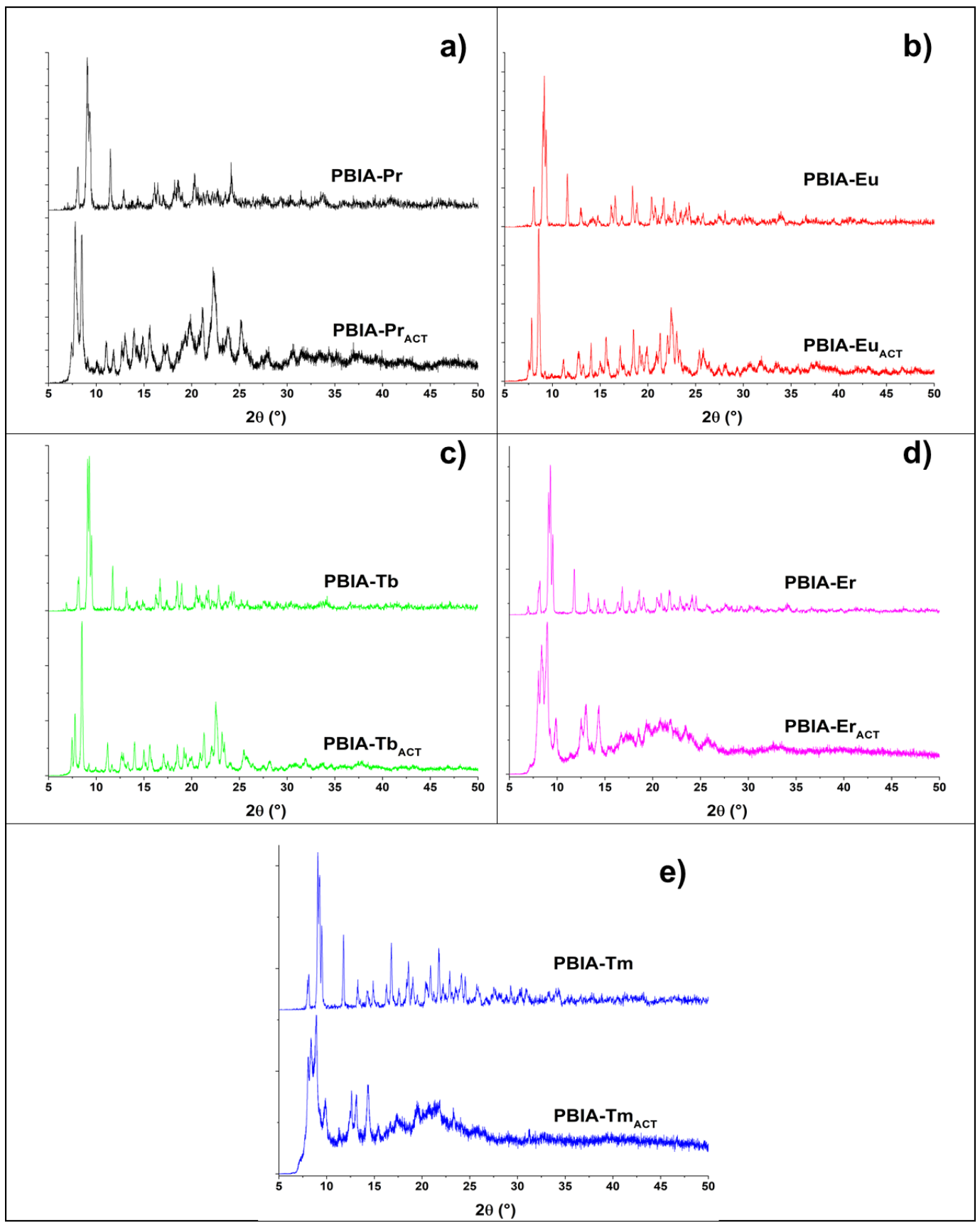

Figure S7. Comparison between X-ray diffractograms of a) PBIA-Pr and PBIA-PrACT; b)

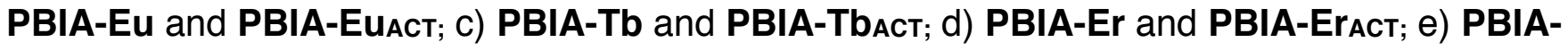
Tm and PBIA-TmACT. 


\section{TGA}

\section{PBIA-Ln (Inactivated materials)}

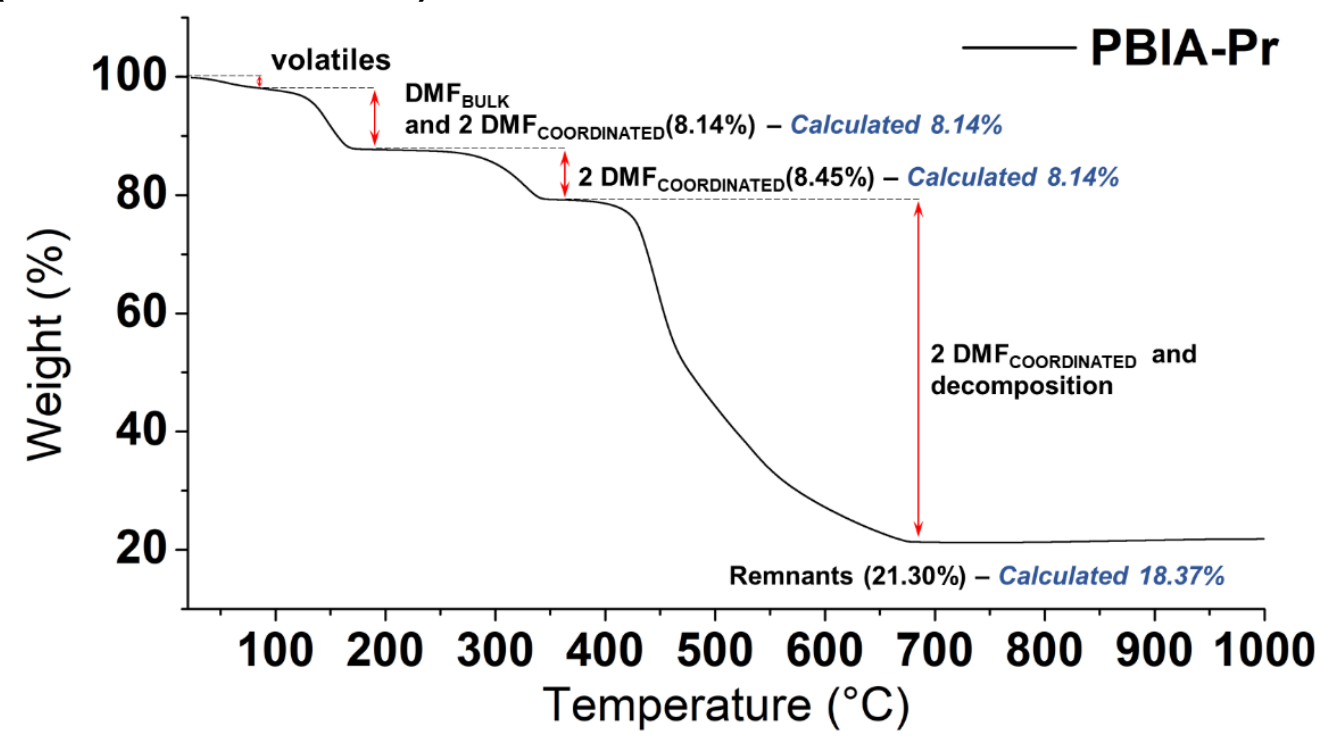

Figure S8. Thermogram of PBIA-Pr obtained in a range from 20 to $995{ }^{\circ} \mathrm{C}$ under $\mathrm{N}_{2}$ flow of 20 $\mathrm{mL} \cdot \mathrm{min}^{-1}$ using a heating rate of $10^{\circ} \mathrm{C} \cdot \mathrm{min}^{-1}$.

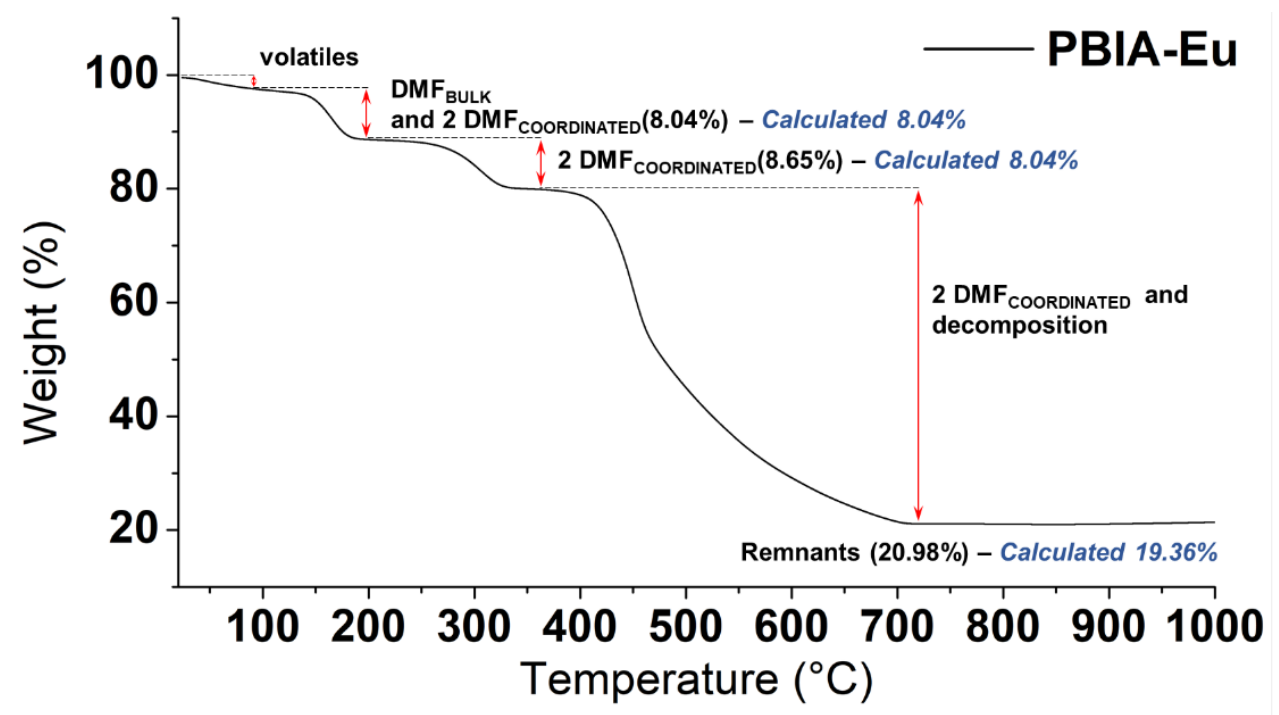

Figure S9. Thermogram of PBIA-Eu obtained in a range from 20 to $995^{\circ} \mathrm{C}$ under $\mathrm{N}_{2}$ flow of 20 $\mathrm{mL} \cdot \mathrm{min}^{-1}$ using a heating rate of $10^{\circ} \mathrm{C} \cdot \mathrm{min}^{-1}$. 


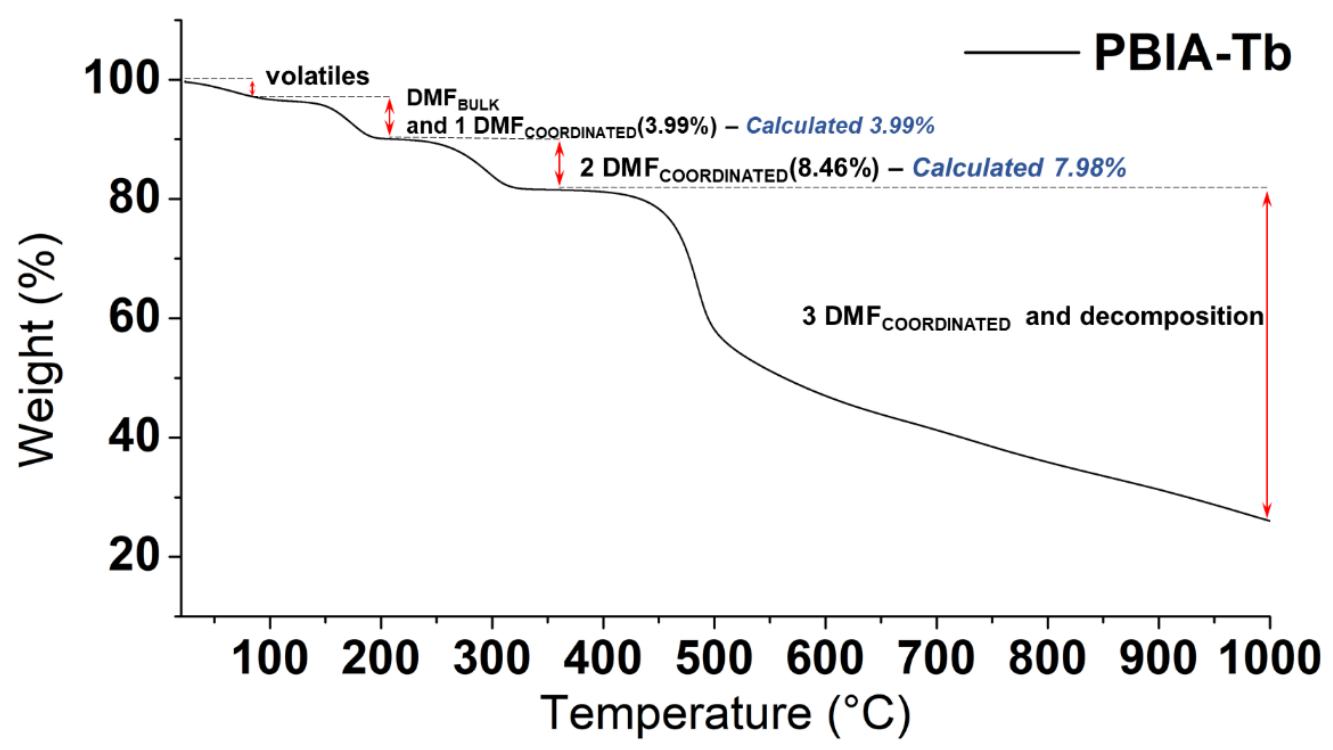

Figure S10. Thermogram of PBIA-Tb obtained in a range from 20 to $995{ }^{\circ} \mathrm{C}$ under $\mathrm{N}_{2}$ flow of $20 \mathrm{~mL} \cdot \mathrm{min}^{-1}$ using a heating rate of $10^{\circ} \mathrm{C} \cdot \mathrm{min}^{-1}$.

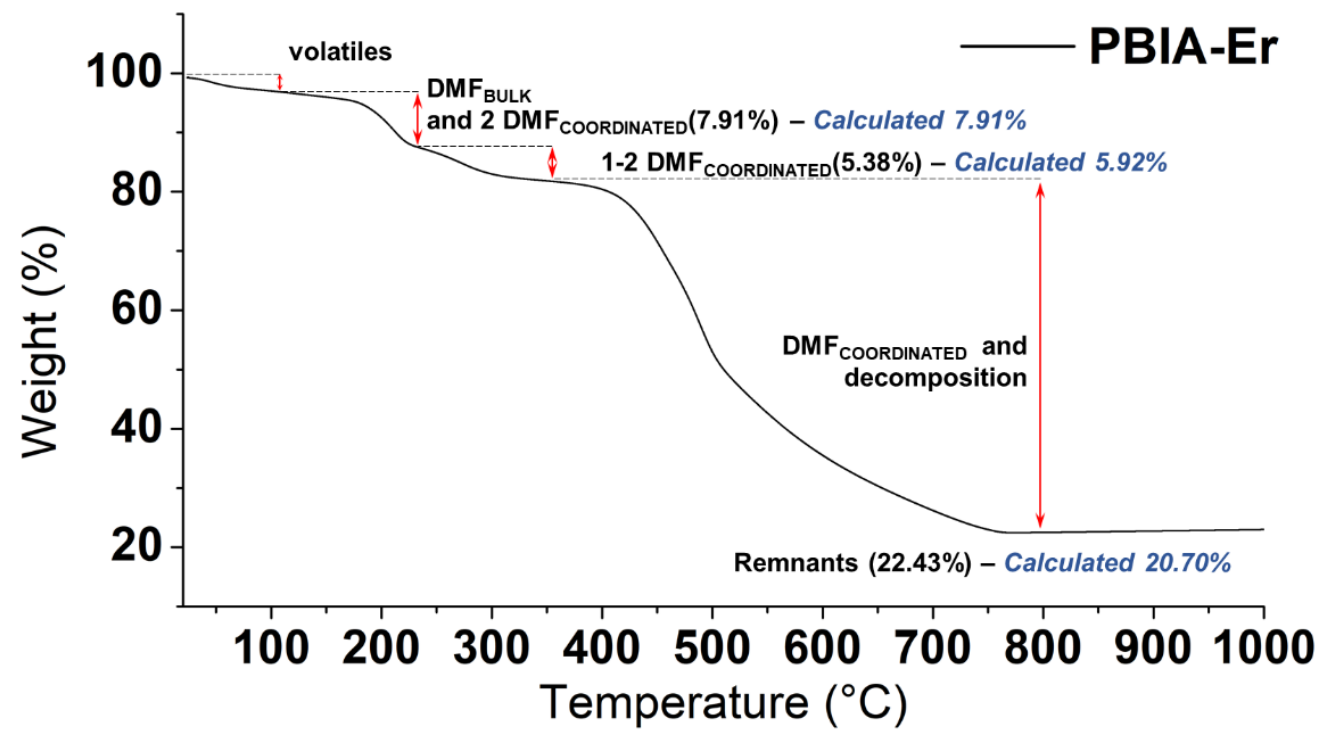

Figure S11. Thermogram of PBIA-Er obtained in a range from 20 to $995^{\circ} \mathrm{C}$ under $\mathrm{N}_{2}$ flow of $20 \mathrm{~mL} \cdot \mathrm{min}^{-1}$ using a heating rate of $10^{\circ} \mathrm{C} \cdot \mathrm{min}^{-1}$. 


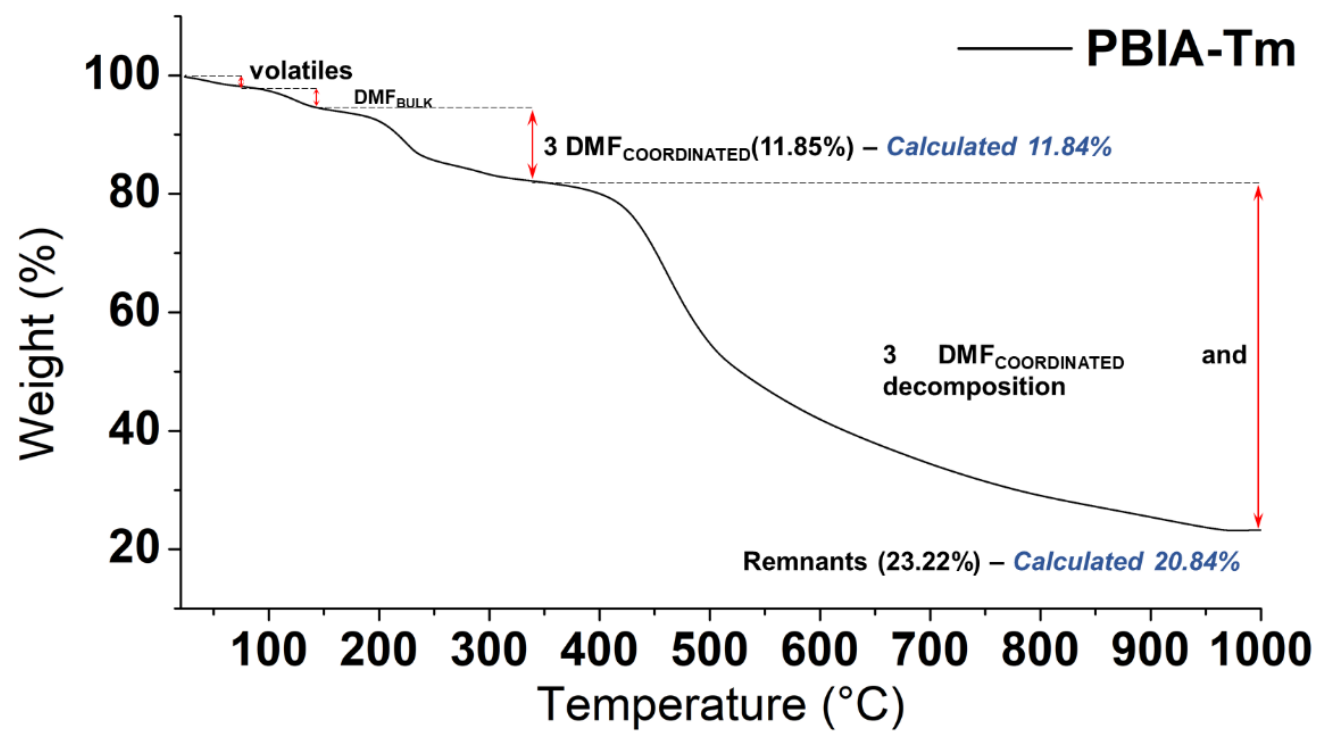

Figure S12. Thermogram of PBIA-Tm obtained in a range from 20 to $995{ }^{\circ} \mathrm{C}$ under $\mathrm{N}_{2}$ flow of $20 \mathrm{~mL} \cdot \mathrm{min}^{-1}$ using a heating rate of $10^{\circ} \mathrm{C} \cdot \mathrm{min}^{-1}$. 
Comparison between activated and inactivated materials

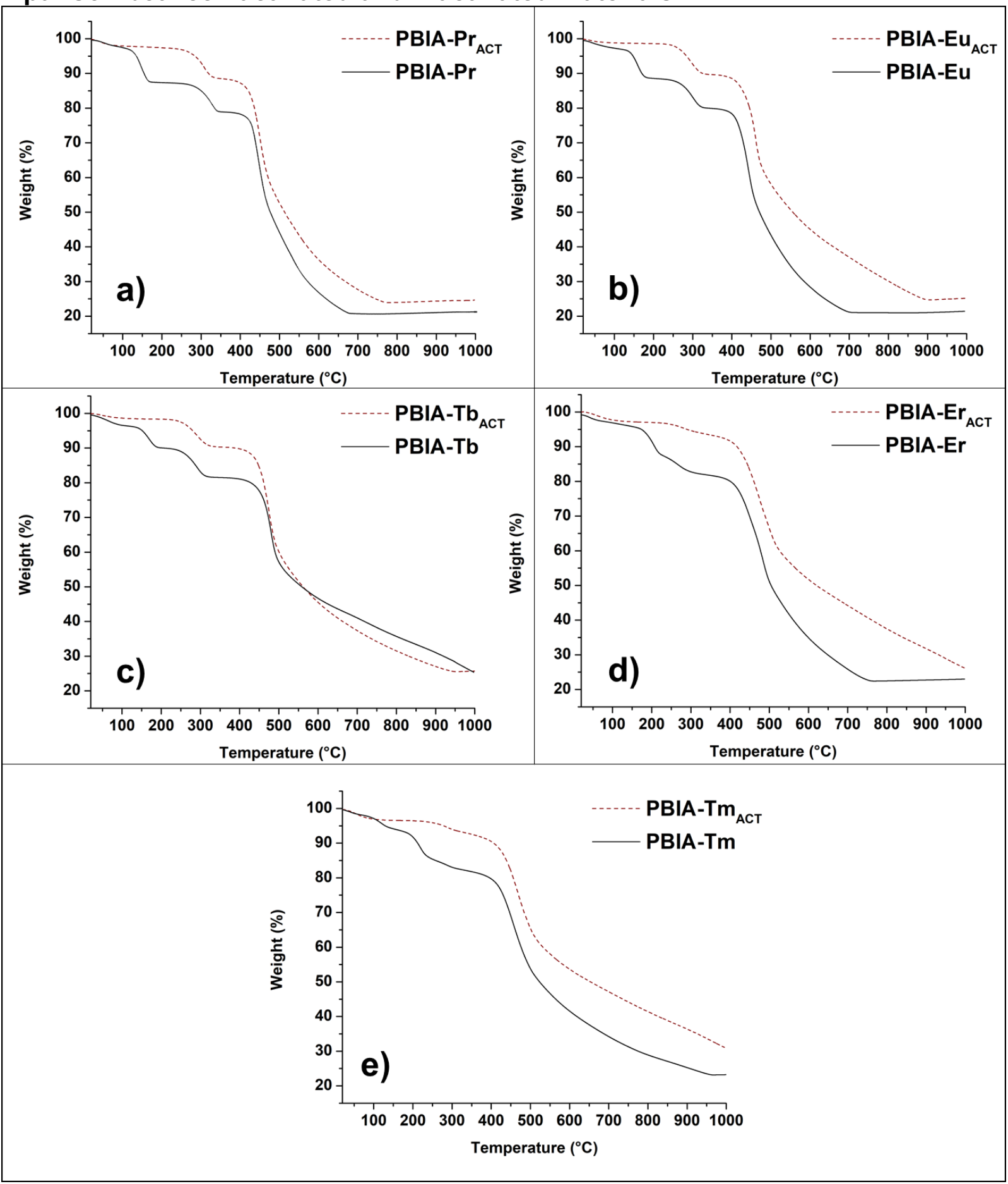

Figure S13. Comparison between the thermograms of a) PBIA-Pr and PBIA-PrACT; b) PBIA-

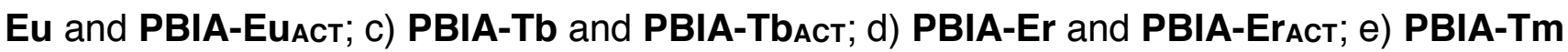
and PBIA-TmACT. 